\title{
An extension of Mazur's theorem on Gateaux differentiability to the class of strongly $\alpha(\cdot)$-paraconvex functions
}

by

\section{S. Rolewicz (Warszawa)}

\begin{abstract}
Let $(X,\|\cdot\|)$ be a separable real Banach space. Let $f$ be a real-valued strongly $\alpha(\cdot)$-paraconvex function defined on an open convex subset $\Omega \subset X$, i.e. such that

$$
f(t x+(1-t) y) \leq t f(x)+(1-t) f(y)+\min [t,(1-t)] \alpha(\|x-y\|) .
$$

Then there is a dense $G_{\delta}$-set $A_{\mathrm{G}} \subset \Omega$ such that $f$ is Gateaux differentiable at every point of $A_{\mathrm{G}}$.
\end{abstract}

Let $(X,\|\cdot\|)$ be a real Banach space. Let $f$ be a real-valued convex continuous function defined on an open convex subset $\Omega \subset X$, i.e.

$$
f(t x+(1-t) y) \leq t f(x)+(1-t) f(y) .
$$

We recall that a set $B \subset \Omega$ of second Baire category is called residual if its complement $\Omega \backslash B$ is of the first Baire category. Mazur (1933) proved that if $X$ is separable, then there is a residual subset $A_{\mathrm{G}}$ such that $f$ is Gateaux differentiable on $A_{\mathrm{G}}$. In this note we extend this result to larger (than convex) classes of functions called strongly $\alpha(\cdot)$-paraconvex functions.

Let $\alpha:[0, \infty) \rightarrow[0, \infty)$ be a nondecreasing continuous function such that

$$
\lim _{t \downarrow 0} \frac{\alpha(t)}{t}=0 .
$$

Let, as before, $(X,\|\cdot\|)$ be a real Banach space. Let $f$ be a real-valued continuous function defined on an open convex subset $\Omega \subset X$. We say that $f$ is $\alpha(\cdot)$-paraconvex if for all $x, y \in \Omega$ and $0 \leq t \leq 1$,

$$
f(t x+(1-t) y) \leq t f(x)+(1-t) f(y)+\alpha(\|x-y\|) .
$$

For $\alpha(t)=t^{2}$ this definition was introduced in Rolewicz (1979a) and the $t^{2}$-paraconvex functions were called simply paraconvex. In Rolewicz (1979b)

2000 Mathematics Subject Classification: Primary 46G05.

Key words and phrases: Gateaux differentiability, strongly $\alpha(\cdot)$-paraconvex functions. 
the notion was extended to the case of $\alpha(t)=t^{\gamma}, 1 \leq \gamma \leq 2$, and the $t^{\gamma}$-paraconvex functions were called $\gamma$-paraconvex.

We say that $f$ is strongly $\alpha(\cdot)$-paraconvex if for all $x, y \in \Omega$ and $0 \leq t \leq 1$,

$$
f(t x+(1-t) y) \leq t f(x)+(1-t) f(y)+\min [t,(1-t)] \alpha(\|x-y\|) .
$$

Of course every strongly $\alpha(\cdot)$-paraconvex function is also $\alpha(\cdot) / 2$-paraconvex. The converse is not true and the conditions warranting the existence $C_{\alpha}$ such that each $\alpha(\cdot)$-paraconvex is strongly $C_{\alpha} \alpha(\cdot)$-paraconvex can be found in Rolewicz (2000). In particular the function $t^{\gamma}, 1<\gamma \leq 2$, satisfies these conditions.

The notion of $\alpha(\cdot)$-paraconvex functions can be treated as a uniformization of the notion of approximate convex functions introduced in the papers of Luc, Ngai and Théra (1999), (2000). We recall that a real-valued function $f$ defined on a convex set $\Omega \subset X$ is called approximate convex if for any $x_{0} \in \Omega$ and $\varepsilon>0$ there is $\delta=\delta\left(\varepsilon, x_{0}\right)$ such that for $x, y$ with $\left\|x-x_{0}\right\|<\delta$ and $\left\|y-x_{0}\right\|<\delta$ and $0 \leq t \leq 1$ we have

$$
f(t x+(1-t) y) \leq t f(x)+(1-t) f(y)+\varepsilon \min [t,(1-t)]\|x-y\| .
$$

We say that a real-valued function $f$ defined on a convex set $\Omega \subset X$ is called uniformly approximate convex if for any $\varepsilon>0$ there is $\delta=\delta(\varepsilon)$ such that (5) holds for $x, y$ with $\|x-y\|<\delta$.

It is easy to show that a real-valued continuous function $f$ is uniformly approximate convex if and only if there is $\alpha(\cdot)$ satisfying $(2)$ such that $f$ is strongly $\alpha(\cdot)$-paraconvex (Rolewicz (2001b)).

We now recall the notion of directional derivative.

By the directional derivative of a continuous function $f$ at a point $x_{0}$ in direction $h$ we mean the number

$$
\left.d^{+} f\right|_{x_{0}}(h)=\lim _{t \downarrow 0} \frac{f\left(x_{0}+t h\right)-f\left(x_{0}\right)}{t} .
$$

It is easy to see that a strongly $\alpha(\cdot)$-paraconvex function has a directional derivative at any point in any direction (Rolewicz (2005)).

We shall show

Proposition 1. Let $\Omega$ be an open convex set in a Banach space $X$. Let $f: \Omega \rightarrow \mathbb{R}$ be an $\alpha(\cdot)$-paraconvex function. Then for any point $x_{0} \in \Omega$ the directional derivative $\left.d^{+} f\right|_{x_{0}}(h)$ is a sublinear (i.e. positively homogeneous and subadditive) function of the direction $h$.

Proof. Positive homogeneity is trivial. Now we shall show subadditivity. Indeed, since $f$ is $\alpha(\cdot)$-paraconvex, for $h_{1}, h_{2} \in X$ and sufficiently small $t$ we 
have

$$
\begin{aligned}
\frac{f\left(x_{0}+t \frac{h_{1}+h_{2}}{2}\right)-f\left(x_{0}\right)}{t} \leq & \frac{1}{2} \frac{f\left(x_{0}+t h_{1}\right)-f\left(x_{0}\right)}{t}+\frac{1}{2} \frac{f\left(x_{0}+t h_{2}\right)-f\left(x_{0}\right)}{t} \\
& +\frac{\alpha\left(t\left\|h_{1}-h_{2}\right\|\right)}{t} .
\end{aligned}
$$

Thus multiplying by 2 and letting $t \rightarrow 0$, by (2) and positive homogeneity of $\left.d^{+} f\right|_{x_{0}}(h)$ we get the triangle inequality

$$
\left.d^{+} f\right|_{x_{0}}\left(h_{1}+h_{2}\right) \leq\left. d^{+} f\right|_{x_{0}}\left(h_{1}\right)+\left.d^{+} f\right|_{x_{0}}\left(h_{2}\right) .
$$

It is easy to observe that a sublinear function is linear if and only if it is homogeneous, i.e. $p(-h)=-p(h)$.

Recall that a strongly $\alpha(\cdot)$-paraconvex function is always locally Lipschitz (Rolewicz (2000)). Basing on this fact it is not difficult to prove that $\left.d^{+} f\right|_{x_{0}}(h)$ is also a locally Lipschitz function.

Any continuous linear functional $x^{*} \in X^{*}$ such that $x^{*}(h) \leq\left. d^{+} f\right|_{x_{0}}$ is called an approximate subgradient of $f$ at $x_{0}$ (see Ioffe (1984), (1986), (1989), (1990), (2000), Mordukhovich (1976), (1980), (1988)). The set of all approximate subgradients of $f$ at $x_{0}$ will be called the approximate subdifferential of $f$ at $x_{0}$ and denoted, as in the classical case, by $\left.\partial f\right|_{x_{0}}$.

It is easy to see that if $\left.\partial f\right|_{x_{0}}$ consists of one functional, $\left.\partial f\right|_{x_{0}}=\left\{x^{*}\right\}$, then $x^{*}$ is a continuous linear functional. Since in this case $\left.\partial f\right|_{x_{0}}(-h)$ $=-\left.\partial f\right|_{x_{0}}(h)$, the function $f$ has Gateaux differential at $x_{0}$, i.e. the limit $\lim _{t \rightarrow 0}\left(f\left(x_{0}+t h\right)-f\left(x_{0}\right)\right) / t$ exists and is equal to $x^{*}(h)$.

A linear functional $x^{*} \in X^{*}$ such that

$$
f(x+h)-f(x) \geq x^{*}(h)-\alpha(\|h\|)
$$

is called a uniform approximate subgradient of $f$ at $x$ with modulus $\alpha(\cdot)$ (or briefly an $\alpha(\cdot)$-subgradient of $f$ at $x)$. The set of all $\alpha(\cdot)$-subgradients of $f$ at $x$ will be called the $\alpha(\cdot)$-subdifferential of $f$ at $x$ and denoted by $\left.\partial_{\alpha} f\right|_{x}$.

The relation between $\alpha(\cdot)$-subdifferentials and directional subdifferentials for strongly $\alpha(\cdot)$-paraconvex function is given by

Proposition 2 (Rolewicz (2001)). Let $\Omega$ be an open convex set in a Banach space $X$. Let $f: \Omega \rightarrow \mathbb{R}$ be a strongly $\alpha(\cdot)$-paraconvex function. Then its $\alpha(\cdot)$-subdifferential is equal to the directional subdifferential, $\left.\partial_{\alpha} f\right|_{x}=\left.\partial f\right|_{x}$

As a consequence we obtain:

Corollary 3. Let $\Omega$ be an open convex set in a Banach space $X$. Let $f: \Omega \rightarrow \mathbb{R}$ be a strongly $\alpha(\cdot)$-paraconvex function. Then $f$ is Gateaux differentiable at $x_{0}$ if and only if its $\alpha(\cdot)$-subdifferential at $x_{0}$ consists of one functional, $\left.\partial_{\alpha} f\right|_{x_{0}}=\left\{x_{0}^{*}\right\}$. 
Basing on this fact we are able to prove the following extension of the classical Mazur theorem (Mazur (1933)):

THEOREM 4. Let $\Omega$ be an open convex set in a separable Banach space $X$. Let $f: \Omega \rightarrow \mathbb{R}$ be a strongly $\alpha(\cdot)$-paraconvex function. Then there is a dense $G_{\delta}$-set $A_{\mathrm{G}} \subset \Omega$ such that $f$ is Gateaux differentiable at every point of $A_{\mathrm{G}}$.

The proof is based on the following

Lemma 5. Let $\Omega$ be an open convex set in a Banach space $X$. Let $f: \Omega \rightarrow \mathbb{R}$ be a strongly $\alpha(\cdot)$-paraconvex function. Then the multifunction $\left.\partial_{\alpha} f\right|_{x}: X \rightarrow 2^{X^{*}}$ is upper semicontinuous from $X$ with the norm topology into $X^{*}$ with the weak ${ }^{*}$ topology. In other words, if $x_{n} \rightarrow x$ and $\left.x_{n}^{*} \in \partial_{\alpha} f\right|_{x_{n}}$ is weak ${ }^{*}$-convergent to $x_{0}^{*}$ then $\left.x_{0}^{*} \in \partial_{\alpha} f\right|_{x_{0}}$.

Proof. Since $f$ is locally Lipschitz, the $\alpha(\cdot)$-subdifferentials $\left.\partial_{\alpha} f\right|_{x_{n}}$ are uniformly bounded, i.e. there is $M>0$ such that $\left\|z^{*}\right\| \leq M$ for any $z^{*} \in$ $\left.\bigcup_{n} \partial_{\alpha} f\right|_{x_{n}}$. Thus

$$
\begin{aligned}
\left|x_{n}^{*}\left(x_{n}\right)-x_{0}^{*}\left(x_{0}\right)\right| & \leq\left|x_{n}^{*}\left(x_{n}\right)-x_{n}^{*}\left(x_{0}\right)\right|+\left|x_{n}^{*}\left(x_{0}\right)-x_{0}^{*}\left(x_{0}\right)\right| \\
& \leq M \| x_{n}-x_{0}|+| x_{n}^{*}\left(x_{0}\right)-x_{0}^{*}\left(x_{0}\right) \mid \rightarrow 0 .
\end{aligned}
$$

Take now an arbitrary $z \in X$. Then

$$
\begin{aligned}
\left\langle x_{0}^{*}, z-x_{0}\right\rangle & =\lim _{t \rightarrow \infty}\left\langle x_{n}^{*}, z-x_{n}\right\rangle \leq \lim _{t \rightarrow \infty}\left[f(z)-f\left(x_{n}\right)-\alpha\left(\left\|x_{n}-z\right\|\right)\right] \\
& =f(z)-f\left(x_{0}\right)-\alpha\left(\left\|x_{0}-z\right\|\right)
\end{aligned}
$$

i.e. $\left.x_{0}^{*} \in \partial_{\alpha} f\right|_{x_{0}}$.

Proof of Theorem 4. Let $\left\{r_{n}\right\}$ be a dense set in the unit ball of $X$. Let $A_{m, n}, n, m=1,2, \ldots$, denote the set of $x \in \Omega$ such that there are $x^{*},\left.y^{*} \in \partial_{\alpha} f\right|_{x}$ such that

$$
\left\langle x^{*}-y^{*}, r_{n}\right\rangle \geq 1 / m \text {. }
$$

By Corollary 3 and the density of $\left\{r_{n}\right\}$ in the unit ball we see that $f$ is Gateaux differentiable at $x_{0}$ if and only $x_{0} \notin \bigcup_{n, m=1}^{\infty} A_{n, m}$.

We shall show that for any $n, m$ the sets $A_{n, m}$ are closed. Indeed, let $\left\{x_{n}\right\}$ be a sequence of elements of $A_{n, m}$ tending to $x_{0} \in \Omega$. By the definition of $A_{m, n}$ there are $x_{n}^{*},\left.y_{n}^{*} \in \partial_{\alpha} f\right|_{x_{n}}$ such that

$$
\left\langle x_{n}^{*}-y_{n}^{*}, r_{n}\right\rangle \geq 1 / m \text {. }
$$

The space $X$ is separable. Thus closed balls are weak*-compact. Therefore we can find subsequences $\left\{x_{n_{k}}^{*}\right\},\left\{y_{n_{k}}^{*}\right\}$ weak ${ }^{*}$-convergent to $x_{0}^{*}, y_{0}^{*}$ respectively. By Lemma $5, x_{0}^{*},\left.y_{0}^{*} \in \partial_{\alpha} f\right|_{x_{0}}$. Passing to the limit in (11) we get

$$
\left\langle x_{0}^{*}-y_{0}^{*}, r_{n}\right\rangle \geq 1 / m
$$

and by the definition $x_{0} \in A_{n, m}$. 
Next observe that the sets $A_{n, m}$ are nowhere dense. Indeed, suppose to the contrary that there is an open set $U \subset \Omega$ such that $U \subset \bar{A}_{n, m}$ $=A_{n, m}$. Take any $\widehat{x} \in U$ and take a line $L_{n}(\widehat{x})=\left\{\widehat{x}+t r_{n} \mid-\infty<t<\infty\right\}$. The function $f$ restricted to $L_{n}(\widehat{x}) \cap \Omega$ is strongly $\alpha(\cdot)$-paraconvex. Thus it is Fréchet differentiable on a residual set (Rolewicz (2002)). Therefore we obtain a contradiction with the fact that $U \subset A_{n, m}$.

Since the sets $A_{n, m}$ are nowhere dense and closed the function $f$ is Gateaux differentiable on a dense $G_{\delta}$-set.

There are non-separable Banach spaces $C(T)$ in which the norms are not Gateaux differentiable at any point (Coban and Kenderov (1985)). Phelps (1989) showed that the function $p(x)=\lim \sup _{n}\left|x_{n}\right|$ defined on the space $\ell^{\infty}$ has this property. There is, however, a class of non-separable Banach spaces in which every convex function is Gateaux differentiable on a dense $G_{\delta}$-set. It is the class of weakly compactly generated spaces (Phelps (1989)). We recall that a Banach space $X$ is weakly compactly generated if there is a weakly compact set $K \subset X$ whose linear span is dense in $X$. Thus there is a natural question:

Problem 5. Let $X$ be a weakly compactly generated Banach space, and let $\Omega \subset X$ be a convex open set. Let $f: \Omega \rightarrow \mathbb{R}$ be a strongly $\alpha(\cdot)$-paraconvex function. Is $f$ Gateaux differentiable on a dense $G_{\delta}$-set?

\section{References}

M. Coban and P. S. Kenderov (1985), Dense Gateaux differentiability of the sup-norm in $C(T)$ and the topological properties of T, C. R. Bulg. Acad. Sci. 38, 1603-1604.

A. D. Ioffe (1984), Approximate subdifferentials and applications I, Trans. Amer. Math. Soc. 281, 389-416.

A. D. Ioffe (1986), Approximate subdifferentials and applications II, Mathematika 33, 111-128.

A. D. Ioffe (1989), Approximate subdifferentials and applications III, ibid. 36, 1-38.

A. D. Ioffe (1990), Proximal analysis and approximate subdifferentials, J. London Math. Soc. $41,175-192$.

A. D. Ioffe (2000), Metric regularity and subdifferential calculus, Uspekhi Mat. Nauk 55, no. 3, 104-162 (in Russian).

D. T. Luc, H. V. Ngai and M. Théra (1999), On $\varepsilon$-convexity and $\varepsilon$-monotonicity, in: Calculus of Variations and Differential Equations, A. Ioffe, S. Reich and I. Shapiro (eds.), Chapman \& Hall, 82-100.

D. T. Luc, H. V. Ngai and M. Théra (2000), Approximate convex functions, J. Nonlinear Convex Anal. 1, 155-176.

S. Mazur (1933), Über konvexe Mengen in linearen normierten Räumen, Studia Math. 4, $70-84$.

B. S. Mordukhovich (1976), Maximum principle in the optimal control problems with nonsmooth constraints, Prikl. Mat. Mekh. 40, 1014-1023 (in Russian). 
B. S. Mordukhovich (1980), Metric approximations and necessary optimality conditions for general classes of nonsmooth extremal problems, Dokl. Akad. Nauk SSSR 254, 1072-1076 (in Russian): English transl.: Soviet Math. Dokl. 22, 526-530.

B. S. Mordukhovich (1988), Approximation Methods in Problems of Optimization and Control, Nauka, Moscow (in Russian).

R. R. Phelps (1989), Convex Functions, Monotone Operators and Differentiability, Lecture Notes in Math. 1364, Springer.

S. Rolewicz (1979a), On paraconvex multifunctions, Oper. Research Verf. (Methods Oper. Res.) 31, 540-546.

S. Rolewicz (1979b), On $\gamma$-paraconvex multifunctions, Math. Japonica 24, 293-300.

S. Rolewicz (2000), On $\alpha(\cdot)$-paraconvex and strongly $\alpha(\cdot)$-paraconvex functions, Control Cybernet. 29, 367-377.

S. Rolewicz (2001a), On the coincidence of some subdifferentials in the class of $\alpha(\cdot)$ paraconvex functions, Optimization 50, 353-360.

S. Rolewicz (2001b), On uniformly approximate convex and strongly $\alpha(\cdot)$-paraconvex functions, Control Cybernet. 30, 323-330.

S. Rolewicz (2002), $\alpha(\cdot)$-monotone multifunctions and differentiability of strongly $\alpha(\cdot)$ paraconvex functions, ibid. 31, 601-619.

S. Rolewicz (2005), Paraconvex analysis, ibid. 34, 951-965.

Institute of Mathematics

Polish Academy of Sciences

Śniadeckich 8

P.O. Box 21

00-956 Warszawa, Poland

E-mail: rolewicz@impan.gov.pl

Received February 2, 2005 